\title{
Free-boundary Ideal MHD Stability of W7-X Divertor Equilibria
}

\author{
C Nührenberg \\ Max-Planck-Institut für Plasmaphysik, 17491 Greifswald, Germany \\ E-mail: Carolin.Nuehrenberg@ipp.mpg.de
}

\begin{abstract}
Plasma configurations describing the stellarator experiment Wendelstein 7-X (W7-X) are computationally established taking into account the geometry of the test-divertor unit and the high-heat-flux divertor which will be installed in the vacuum chamber of the device [Gasparotto M et al., 2014 Fusion Engineering and Design 89 2121]. These plasma equilibria are computationally studied for their global ideal magnetohydrodynamic (MHD) stability properties. Results from the ideal MHD stability code CAS3D [Nührenberg C 1996 Phys. Plasmas 3 2401], stability limits, spatial structures and growth rates are presented for freeboundary perturbations. The work focusses on the exploration of MHD unstable regions of the $\mathrm{W} 7-\mathrm{X}$ configuration space, thereby providing information for future experiments in W7-X aiming at an assessment of the role of ideal MHD in stellarator confinement.
\end{abstract}




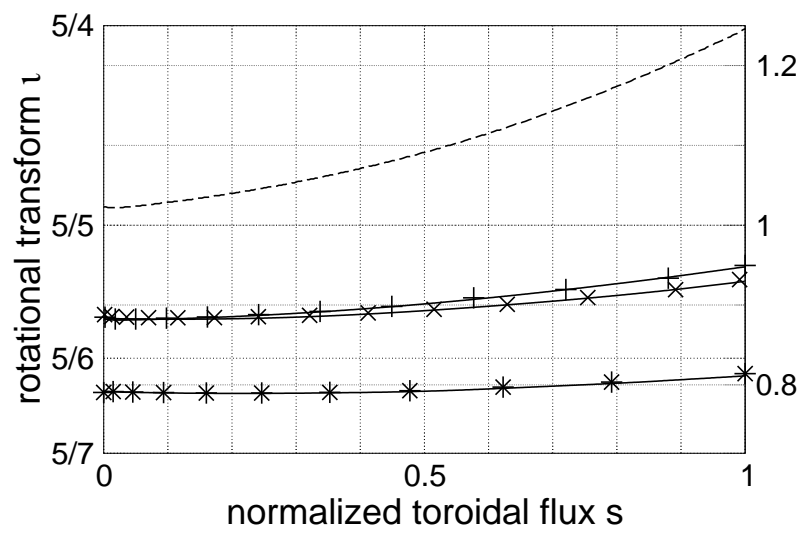

Figure 1. Rotational transform profiles versus normalized toroidal flux for W7-X variants. Standard case: magnetic mirror 0.11 and $\langle\beta\rangle=0.05(\times),\langle\beta\rangle=0.03(+)$; low-iota case: magnetic mirror 0.22 and $\langle\beta\rangle=0.009(*)$. Results from NEMEC are shown as solid lines, results from field-line tracing in the magnetic fields from the NEMEC+EXTENDER_P+VACFIELD procedure as symbols. The vacuum rotational transform for a high-iota case is indicated $(---)$ for comparison only.

\section{Introduction}

In the design of present-day stellarator devices, the implications of ideal magnetohydrodynamic (MHD) stability were of great importance, a configuration being ideal-MHD unstable would not have been considered ready for approval. In experiments, however, several stellarators showed quiescent plasmas in scenarios predicted to be unstable according to ideal MHD theory or close to the MHD stability limit. Examples are very quiescent ECRH-heated plasmas in Wendelstein 7-AS [1], and the conclusion that W7-AS was not limited by pressure-driven MHD instabilities, neither in the achievable plasma pressures nor in the performance of the high-plasma-pressure discharges $[2,3]$, and almost shearless configurations in TJ-II [4]. In the Large Helical Device (LHD), explicitly MHDunstable scenarios were experimentally investigated [5], by making use of the flexibility of its magnetic configuration.

The Wendelstein 7-X stellarator experiment (W7-X) in Greifswald, Germany, has ended its commissioning phase [6] and started the first plasma operation [7]. In its second operational phase, W7-X will be equipped with a high-heat flux divertor $[6,8]$ exploiting the intrinsic island chains in the plasma periphery present for edge rotational transform values of $\iota=5 / 4,5 / 5$, and 5/6. In the MHD context studied here, it is important that an MHD stable plasma performance with plasma pressures yielding $\langle\beta\rangle \approx 0.05$ is one of the design goals of W7-X. Here, $\langle\beta\rangle=2 \mu_{0}\left\langle p / B^{2}\right\rangle$ is the volume averaged ratio of the plasma pressure and the internal magnetic field energy density.

Therefore, this work, which computationally revisits the ideal MHD properties of $\mathrm{W} 7-\mathrm{X}$ plasmas [9], focusses on the study of unstable cases in the W7-X configuration space, possibly providing plasma scenarios in which experiments might assess the role of ideal MHD in stellarator confinement.

Following [10,11], where the ideal MHD displacement vector $\boldsymbol{\xi}$ describes the change in location of a fluid element from its initial position, the energy that is required to perturb a plasma equilibrium is $2 \delta W=-\int_{\text {plasma }} \boldsymbol{\xi} \cdot \mathbf{F}(\boldsymbol{\xi}) \mathrm{d}^{3} r$. Here, the force operator of linearized ideal MHD is $\mathbf{F}(\boldsymbol{\xi}) \equiv \mathbf{j}_{0} \times \mathbf{B}_{1}+\mathbf{j}_{1} \times$ $\mathbf{B}_{0}-\boldsymbol{\nabla} p_{1}$, the subscripts indicating equilibrium (0) and perturbed (1) magnetic field, B, current density, $\mathbf{j}$, and the scalar plasma pressure, $p$. For stellarator equilibria, the ideal MHD energy principle as formulated in $[10,11]$ has often been treated with approximate methods, such as averaging and stellarator expansion $[12,13]$. The true geometry, however, has been used in the TERPSICHORE [14] and CAS3D [15] codes, the latter being used in this work. A Galerkin method, with basis functions combining finite elements for the radial direction and Fourier expansions for the poloidal and toroidal magnetic angles, is implemented for the minimization of the first-order perturbed MHD energies. In the CAS3D code, the full energy principle is employed, thus permitting the study of global, compressible, free-boundary linear ideal MHD modes for their spatial structures and time dependence with growth rates or frequencies.

The paper is organized as follows. In section 2, the equilibrium calculations are described. The stability properties of these W7-X variants are analyzed in section 3. In section 4, a summary and conclusions are given.

\section{Equilibrium calculations for $\mathrm{W} 7-\mathrm{X}$ variants}

The W7-X magnetic-field parameters do not define just a single configuration, but rather a configurational space, thus offering a variety of experimental scenarios. In this work, configurations are explored that fall 


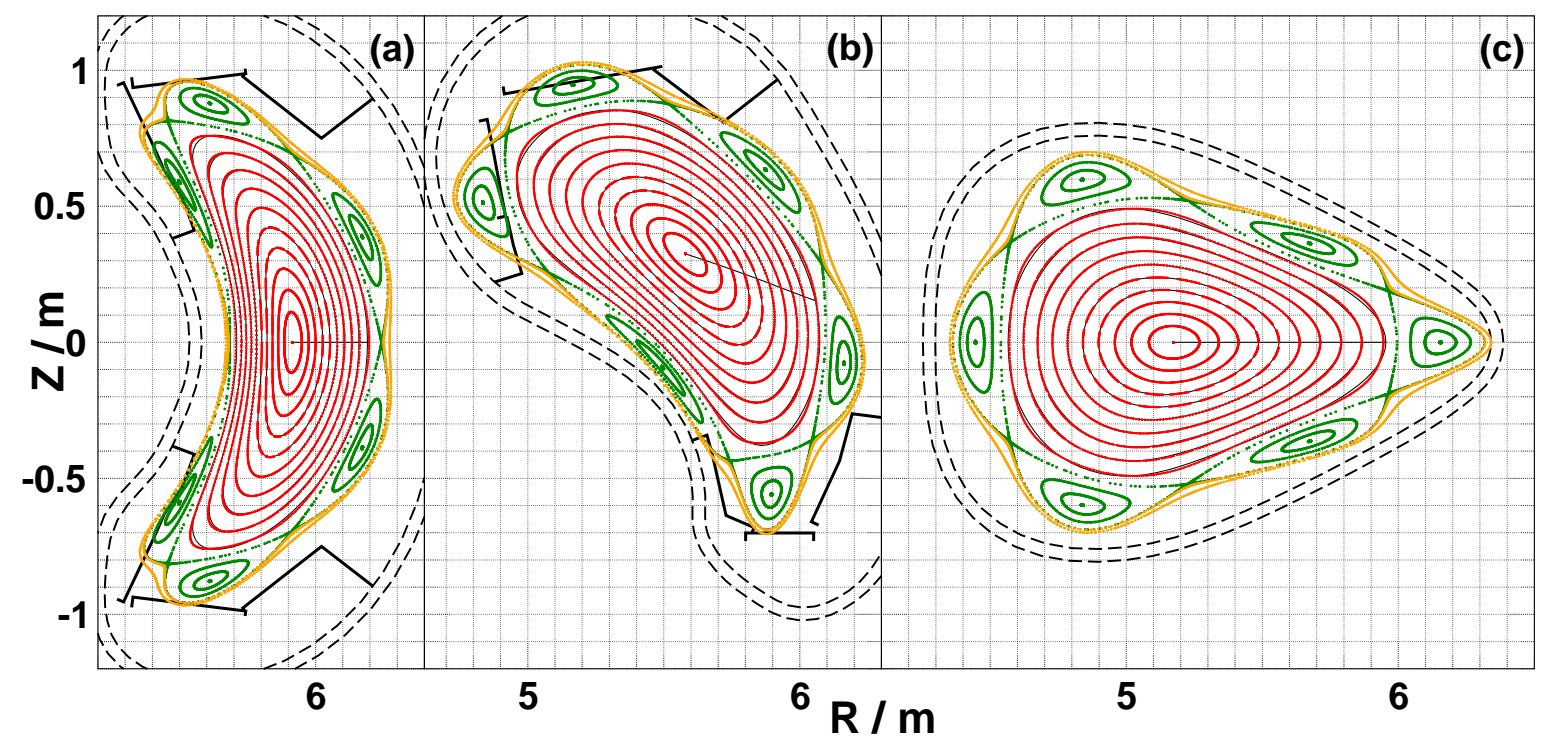

Figure 2. Field-line tracing in the finite- $\beta$ magnetic field of a W7-X low-iota high-mirror variant (plasma volume $V_{\text {plasma }}=27.7 \mathrm{~m}^{3}$, $\langle\beta\rangle=0.009, m_{\text {ratio }}=0.22, \Phi_{\text {edge }}=1.4 \mathrm{Vs}$ ): NEMEC results (thin black lines) are compared with field-line tracing (red dotted inside the plasma, green dotted for the $5 / 6$ island, orange dotted for closed surfaces beyond the island chain). The toroidal cross-sections are: (a) the beginning of a field period $\left(\varphi=0^{\circ}\right)$; (b) near a quarter of a field period $\left(\varphi=16^{\circ}\right)$; (c) at the middle of a field period $\left(\varphi=36^{\circ}\right)$. In each sub-frame, dashed lines indicate the vacuum vessel, thick solid lines show divertor elements.

in two of the three foreseen divertor modes, firstly so-called standard-case plasmas with unity rotational transform, $\iota \lesssim 1$, at the plasma edge and the $5 / 5$ island chains in use for the divertor, and secondly low-iota plasmas with the $5 / 6$ island for the divertor operation. Figure 1 shows the $\iota$-profiles for some of the plasma configurations studied in sections 2 and 3.

Net-current-free plasmas will be studied here. The elimination of the bootstrap current can be accomplished by proper adjustment of the magnetic field with a sufficiently high magnetic mirror [16]. The magnetic miror describes the change of the magnetic field strength $B$, e.g. on the magnetic axis, and may be defined as

$m_{\text {ratio }}=\frac{B_{\varphi=0^{\circ}}-B_{\varphi=36^{\circ}}}{2 B_{\mathrm{av}}}$

with $B$ taken on the magnetic axis at the bean-shaped cross-section, $\varphi=0^{\circ}$, at the triangular cross-section, $\varphi=36^{\circ}$, and averaged along the magnetic axis, $B_{\mathrm{av}}$. In W7-X, $m_{\text {ratio }} \approx 0.1$ is sufficient for vanishing nettoroidal current in the standard case, $m_{\text {ratio }} \approx 0.24$ is needed in the low-iota variant [16].

A suite of codes has been used to obtain the plasma equilibria studied in section 3 . The BiotSavart solver VACFIELD [17] computes the magnetic field from the coil currents of a magnetic confinement device. This magnetic field is used in the ideal MHD equilibrium code VMEC [18] in its free-boundary version NEMEC [19] to compute free-boundary plasma equilibria. The NEMEC code maintains a continuous total pressure across the plasma boundary and simultaneously varies the plasma surface to minimize the plasma energy. In zero-edge-pressure cases, this reduces to $B_{0}^{2}$ continuous at the plasma boundary, with $B_{0}$ the total equilibrium magnetic field.

The equilibrium is determined under the assumption of nested magnetic surfaces, a simplification which appears adequate for most of the subsequent applications, e.g. stability studies. In this picture, magnetic islands forming at the so-called natural resonances do not exist. Instead, a manifestion of rational values of the rotational transform, $\iota=n / m$, are diverging parallel current densities existing if, for a rational $\iota$ inside the plasma, the numerator, $n$, is an integer multiple of the number of identical sections or field periods, $N_{\mathrm{p}}$, of the confinement device, and if the respective field perturbation exists. For a rational $\iota$, the field lines make $n$ poloidal transits and $m$ toroidal transits around the plasma torus before enclosing upon themselves. Flattening the pressure around the resonance eliminates the infinite parallel current density $[20,21]$. To this end, in a NEMEC equilibrium calculation, the equilibrium pressure gradient is constrained to be zero with smooth transitions near a natural resonance, the flux interval chosen just wide enough to ensure stability regarding local stability criteria. A small net toroidal current exists in this equilibrium, because the rotational transform profile is fixed to the one of the neighboring equilibrium without pressure flattening. Such equilibria have been used for global stability analyses in previous work [9] and are examined in this work, 


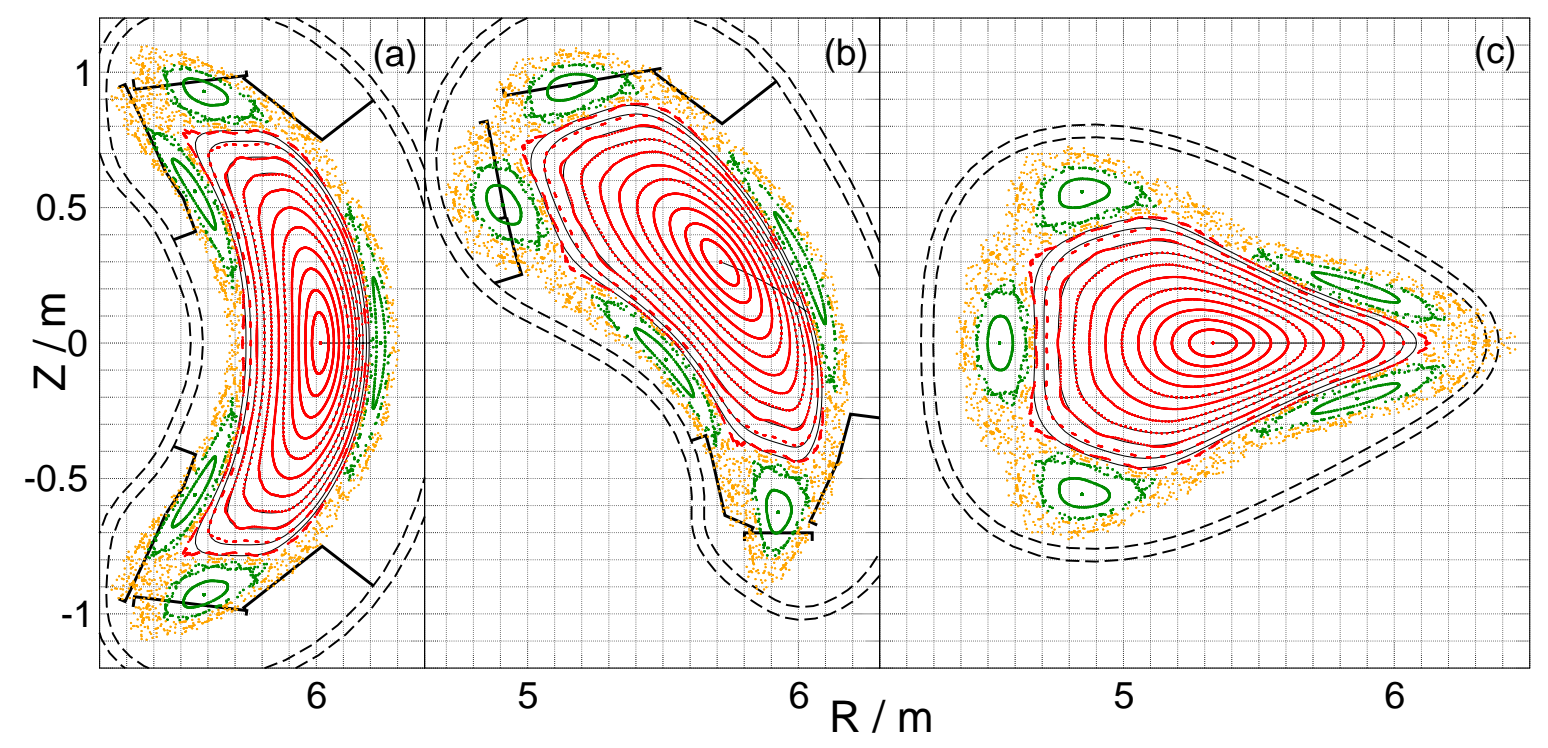

Figure 3. Field-line tracing in the finite- $\beta$ magnetic field of a W7-X standard high-mirror variant (plasma volume $V_{\text {plasma }}=26.2 \mathrm{~m}^{3}$, $\langle\beta\rangle=0.05, m_{\text {ratio }}=0.11, \Phi_{\text {edge }}=1.5 \mathrm{Vs}$ ): NEMEC results (thin black lines) are compared with field-line tracing (red dotted inside the plasma, green dotted for the $5 / 5$ islands, orange dotted for ergodic regions). The toroidal cross-sections are as in figure 2 .

too, section 3.2. If pressure flattening is not invoked, then the resonant components of $j_{0 \|}$ are constrained to be zero, because otherwise any stability calculation, local or global, will only give evidence of the diverging current densities at the natural resonances. Stability results for such equilibria are presented in section 3.2 , too. As an alternative to flattening the pressure profile, one can let the rotational transform $\iota$ make a small jump at the rational surface. This allows the pressure gradient to remain continuous and reflects the known behaviour of nonlinear solutions of the equilibrium equation [22].

The compatibility of the plasma, the surrounding island chains, and, possibly, divertor components is checked by field-line tracing in the finite-plasmapressure magnetic field. For this purpose, the fieldline tracer GOURDON [23] has been augmented with the W7-X divertor geometry. As the equilibrium solver only delivers the total magnetic field in the plasma region and on its boundary, the EXTENDER_P code [24] implementing the virtual casing principle [25] is used to calculate the field of the plasma current in the outside region and the magnetic field produced by the coils in the plasma region. Outside the plasma boundary, the total magnetic field from coil and plasma currents is obtained by superposition. In the plasma region, a test for the quality of the equilibrium calculation is available in the following sense: The virtual casing principle calculation yields the plasmacurrent field by $\mathbf{B}_{\mathrm{pc}}=\mathbf{B}_{\text {NEMEC }}-\mathbf{B}_{\text {EXTENDER }}$. The total magnetic field is obtained by superposing the coils' field $\mathbf{B}_{\text {VACFIELD }}$ from the Biot-Savart solver,
$\mathbf{B}_{\text {total }}=\mathbf{B}_{\mathrm{pc}}+\mathbf{B}_{\text {VACFIELD. }}$ Thus, good agreement is found between the coils' field from the Biot-Savart solver and from the virtual casing principle calculation, if field-line tracing in $\mathbf{B}_{\text {total }}$ maps out the magnetic surfaces determined by the equilibrium solver. Since the VMEC code exploits the assumption of nested magnetic surfaces, the quality of the geometry of the free-boundary equilibrium, notably of the plasma boundary, can be improved if this field-line tracing shows islands in the plasma region. Small remnants of islands in the plasma region indicate a slight imperfection of the NEMEC calculation.

In the free-boundary equilibrium calculation with NEMEC, the toroidal magnetic flux enclosed by the plasma edge, $\Phi_{\text {edge }}$, is one of the input parameters, the plasma volume being proportional to it, $V_{\text {plasma }} \propto$ $\Phi_{\text {edge }}$. The iterative adjustment of $\Phi_{\text {edge }}$ introduced in [26] and applied in [24] is used here, too.

A trade-off has to be made between the maximum calculable plasma volume and the magnetic-field structure near the so-called $x$-points of the edge islands' separatrices or near the possibly ergodic edge region. The Fourier representations that the NEMEC code uses for the magnetic surfaces are not well adapted to the geometrically complicated situation near the x-points and would require the inclusion of poloidal Fourier indices $m>20$, which leads to a deterioration of the equilibrium code's convergence properties. Therefore, in the equilibria presented here, the plasma boundary as obtained from the iterative adjustment of $\Phi_{\text {edge }}$ may still be surrounded by a very thin layer of closed magnetic surfaces in the vacuum region, which are too 


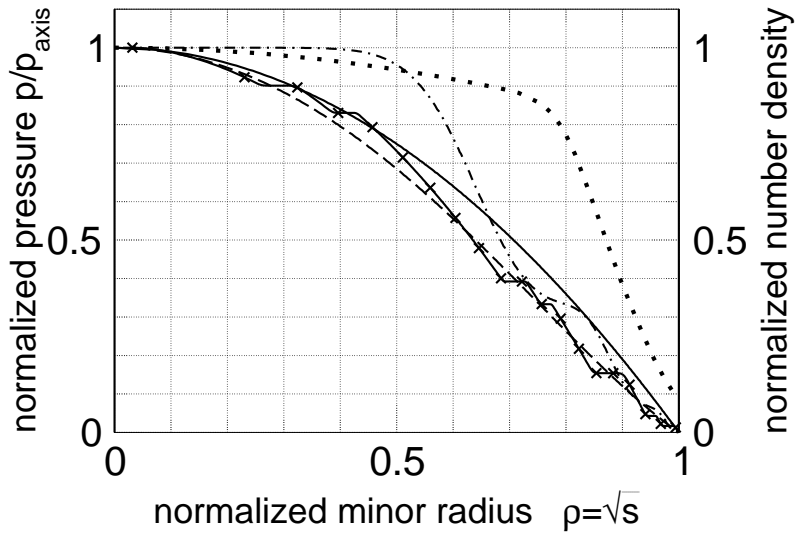

Figure 4. Equilibrium pressure profiles, $p_{0} / p_{0}(0)$, used in the NEMEC calculations. Linear in normalized toroidal flux (and $\times$ with flat-pressure regions), bell-shaped in normalized toroidal flux $(---)$, from transport calculation ( $-\cdot-)$. The normalized equilibrium mass density $\rho_{0} / \rho_{0}(0)$ used in the CAS3D calculations is shown, too $(\cdots \cdots)$.

demanding geometrically to be modeled by the NEMEC code. In the case of more strongly ergodized islands, however, a stochastic layer surrounds the plasma.

The NEMEC computations were done with up to 700 flux intervals, on 72 toroidal planes per field period, and with 90 poloidal points. The Fourier expansions used 18 (9 or 12) poloidal (toroidal) Fourier harmonics.

The coil currents define the magnetic topology and topography. The main components of the W7-X magnet system are fifty modular and twenty planar coils with five modular and two planar coil types because of the five-fold periodicity and the stellarator symmetry of the machine. The planar coils can be used to adjust the rotational transform, $\iota$, near the plasma boundary. For the W7-X low-iota variant, the corresponding coil-current distribution was determined by an optimization in a 6 -dimensional space targeting several properties of the vacuum magnetic field. They are the vacuum-field magnetic well (or hill, the definition is given in section 3), the location of the island-chain o-points, the magnetic mirror of (1), the values of the rotational transform on the magnetic axis and near the plasma boundary, and the magnetic axis position. For the standard case the adjustment was found iteratively. The current loads for the cases studied are summarized in table A1.

In the W7-X low-iota case shown in figure 2, the magnetic field mirror on the magnetic axis is $m_{\text {ratio }}=0.22$ for small plasma pressure, $\langle\beta\rangle=0.009$ and a pressure profile $(-.-$ in figure 4$)$ resulting from a transport calculation $[16,27]$ for a highdensity-O2-mode heating scenario. The plasma volume is $V_{\text {plasma }}=27.7 \mathrm{~m}^{3}$. The field-line tracing in the finite- $\beta$ magnetic field shows that the plasma, red points in figure 2, does not touch the divertor structures. In the outer region, the field-line tracing was done without the divertor plates so that the edgemagnetic-field structure can be studied. The nearly non-ergodized $\iota=5 / 6$ island tube with six cuts on each meridional cross-section is surrounded by closed magnetic surfaces.

Three representative cross-sections for the W7-X standard high-mirror case are shown in figure 3 , with the intersection points from the field-line tracing in red inside the plasma, in green for the $5 / 5$ islands, and in orange for ergodic regions. For this equilibrium with $\langle\beta\rangle=0.05, m_{\text {ratio }}=0.11$, and $\Phi_{\text {edge }}=1.5 \mathrm{Vs}$, a pressure profile linear in the normalized toroidal flux coordinate $s$ was used in the NEMEC computation, which is flattened at the natural resonances (solid line with $\times$ in figure 4), e.g. $\iota=10 / 11$ near $\rho=\sqrt{s}=0.87$. The low-shear rotational transform varies between $\iota(0)=0.884$ and $\iota(1)=0.93$, marked with $\times$ in figure 1. The plasma volume is $V_{\text {plasma }}=26.2 \mathrm{~m}^{3}$.

If $\iota_{\text {edge }}=5 / 5$, there are five individual island tubes winding around the plasma column. In that, the magnetic topology differs from the one with $\iota_{\text {edge }}=5 / 6$, figure 2 , where there is a single island in the peripheral region. With increasing plasma pressure the islands become larger and more ergodized, see figure 3 for $\langle\beta\rangle=0.05$.

\section{Global MHD stability of W7-X variants}

In the optimization procedure [20] leading to the definition of the W7-X configurational space [28], good MHD stability properties were one of seven optimization principles. They were targeted by means of either evaluating in the case of the local Mercier $[29,30]$ and peeling $[30,31]$ stability criteria, or by their driving terms as proxies for the case of the local ballooning analysis [30,32]. Here, the local stability is used in a supplementary way only, since, in the ideal MHD framework, the global mode analysis gives a closer description of the plasma properties, e.g. determining the poloidal and toroidal perturbation structures and growth rates or frequencies, which is of importance in the interpretation of experimental results.

The magnetic well is a figure of merit in ideal MHD considerations. With the contained volume $\int V^{\prime} \mathrm{d} s$, the magnetic well can be defined [10] as $V^{\prime \prime}<0$. In the W7-X configuration space, a vacuumfield magnetic well prevails. The low-iota cases are an exception and have a magnetic hill, $V^{\prime \prime}>0$. As discussed in section 3.1 and for fixed-boundary MHD modes in [9], this unfavourably influences their MHD stability properties. In the Mercier criterion [29,30], the stabilizing effect of the shear and terms scaling 
as the net-toroidal current are small for low-shear net-current-free stellarators. Furthermore, since being weighted by the pressure gradient, which is usually negative, a magnetic hill acts in a destabilizing way, so that, according to the Mercier criterion, the low-iota cases are locally unstable.

In stellarators, the finite number of identical toroidal sections of the magnetic confinement device, the so-called field periods, and the stellarator symmetry, which corresponds to up-down symmetry in an axi-symmetric configuration, lead to the notion of so-called mode families and to the decoupling of odd- and even-parity perturbations $[9,15]$. In the development of a global ideal MHD stability code, e.g. the CAS3D code, the use of these properties helps to limit the size of the computational problem and so renders feasible the study of fine-scale freeboundary perturbation structures which require high resolution. W7-X has a five-fold periodicity, $N_{\mathrm{p}}=5$, and, therefore, three mode families exist, $N=0,1,2$, comprising toroidal Fourier numbers $\pm N \pm N_{\mathrm{p}} k$ for integer $k$. With $s$ the normalized toroidal flux which is used as flux-surface label, the dimensionless scalar normal displacement is $\xi^{s}=\boldsymbol{\xi} \cdot \boldsymbol{\nabla} s$. For even-parity perturbations, it is used with a purely cosine Fourier series. In this and other work based on the CAS3D code, $\alpha_{m n}=2 \pi\left(m \theta+n \phi / N_{\mathrm{p}}\right)$ is used in the definition of a Fourier series, so that in magnetic coordinates the resonance condition derives from

$\mathbf{B}_{0} \cdot \nabla\left[\xi_{m n}^{s} \cos \alpha_{m n}\right] \propto(m \iota+n) \xi_{m n}^{s} \sin \alpha_{m n} \quad$.

In the stability analyses discussed below, all mode families and both mode parities have been employed, yielding results essentially independent of the choice made, which is in keeping with previous findings $[9,15]$. For each of the three scalar perturbation components, the one normal to flux surfaces, $\xi^{s}$, and two contributing to the displacement within flux surfaces, the angle-like variation is described by a Fourier series. In the work described here, the perturbation Fourier tables used 325 harmonics, filling a diagonal band following the resonance condition for $\iota_{\text {edge }}$.

As being the physically relevant boundary condition, all perturbations discussed in this work have been determined using the free-boundary condition. In this scenario, the space of admissible perturbation functions is larger and includes the perturbations of the fixed-boundary case. Perturbations resembling more or less fixed-boundary perturbations in spite of the freeboundary condition, do not change significantly when subjected to the fixed-boundary condition. However, perturbations which are evidently free-boundary, will change considerably when forced to be fixed-boundary, will become less unstable or even stable.

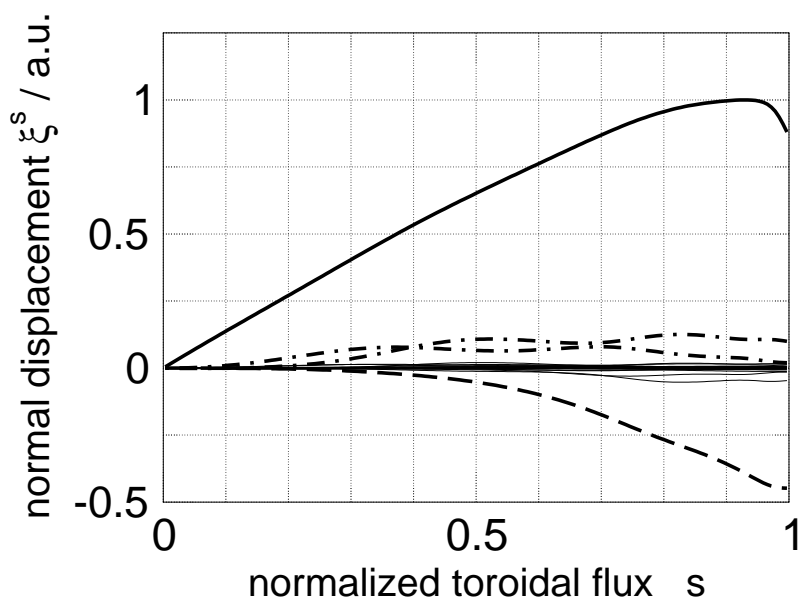

Figure 5. Fourier harmonics of the normal displacement, $\xi^{s}$, versus normalized toroidal flux, $s$, of an unstable perturbation in the low-iota case of figure 2. The dominant harmonic is $m=5$, $n=-4(-)$, with toroidicity-induced side-bands indicated by dot-dashed curves. The most important helical side-band is for $m=11, n=-9(---)$. CAS3D computation parameters: 325 perturbation Fourier harmonics (40 strongest shown), 128 radial points.
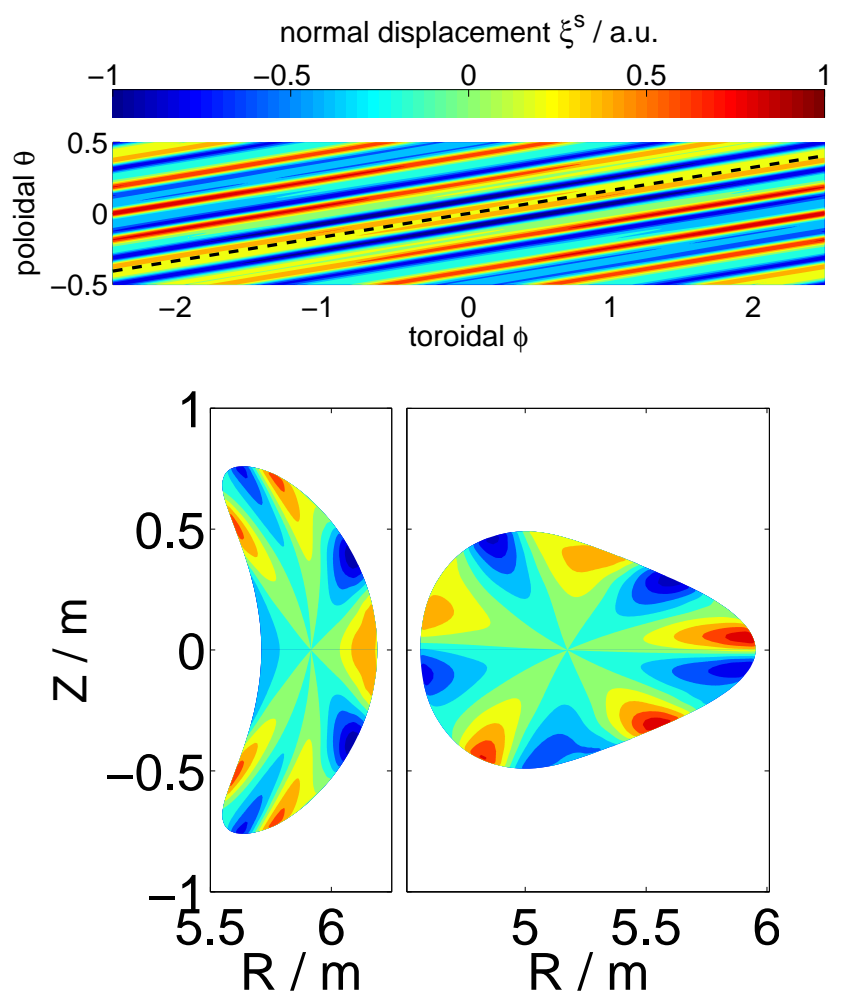

Figure 6. Unstable perturbation in the low-iota case of figures 2 and 5: Contours of the normal displacement, $\xi^{s}$ in magnetic coordinates, on the developed plasma boundary, on the $\varphi=0^{\circ}$ bean-shaped, and on the $\varphi=108^{\circ}$ triangular cross-section. The outside of the torus is near $\theta_{\text {poloidal }}=0$. The beanshaped cross-section is at $\phi_{\text {toroidal }}=0 \pm n$, the triangular one at $\phi_{\text {toroidal }}=0.5 \pm n, n=1,2$. One toroidal transit of the magnetic field-line $(\iota=0.8113)$ is indicated by --- . The colour map applies to all frames. 
A non-uniform equilibrium mass density profile, $\rho_{0}$ in figure 4 , has been used in the kinetic energy related to the MHD displacement, $W_{\text {kin }}=\int_{\text {plasma }} \rho_{0}|\boldsymbol{\xi}|^{2} \mathrm{~d}^{3} r$. It was obtained from transport computations [16,27], and affects growth rates but not stability thresholds.

\subsection{Edge-rotational transform $5 / 6$}

Even at low plasma pressure, the low-iota highmirror configuration is unstable against low-modenumber global perturbations. By way of example, a low-mode-number perturbation which is unstable at $\langle\beta\rangle=0.009$ is shown in figures 5 and 6 . This even-parity perturbation belongs to the $N=1$ mode family. As is typical for so-called Mercier-type perturbations, there is one dominant Fourier harmonic with only small side-bands. The $m=5$ poloidal Fourier contents can be seen in the bottom frames of figure 6 showing the characteristic bean-shaped and triangular cross-sections. The $n=-4$ toroidal Fourier contents becomes evident when counting extrema of the normal displacement along a $\theta=$ const. path on the developed plasma boundary, top frame of figure 6 . This is in keeping with the resonance condition, $m \iota+n=0$, for the low-order rotational transform $\iota_{\text {res }}=4 / 5$ which is present inside the plasma, see figure 1 . In figure 6 , one toroidal transit of a plasma-boundary field line, $\theta=\theta_{0}+\iota \phi / N_{\mathrm{p}}$ in magnetic coordinates, shows that the perturbation is aligned with the magnetic field, i.e. is resonant. The perturbation is evidently free-boundary. On the plasma boundary, neither a significant poloidal nor a significant toroidal variation of the extreme amplitudes is present.

Assuming a hydrogen plasma and a central equilibrium electron number density of $n_{\mathrm{e}}(0)=$ $0.3410^{20} / \mathrm{m}^{3}[16,27]$, the $e$-folding time,

$\tau_{e-\text { fold }}=\sqrt{\frac{\mu_{0} \rho_{0}(0)}{\left|\lambda_{\mathrm{CAS} 3 \mathrm{D}}\right|}}$,

is $\tau_{e-\text { fold }} \approx 13 \mu$ s for this perturbation. Figures 5 and 6 show a low-mode number perturbation being spatially extended as is typical for low-shear stellarators. CAS3D stability calculations demonstrate that perturbations with the dominant harmonic satisfying the same resonance condition but at higher multiplicity are more unstable, and, the higher their mode numbers, the stronger their radial localization around the respective resonant surface. By way of example, a series of perturbations is described, each with the largest harmonic being resonant at $\iota=4 / 5$ at roughly $90 \%$ of the normalized minor radius in the low-iota $\mathrm{W} 7-\mathrm{X}$ case considered here. For each of the free-boundary perturbations, the ratio of the maximum amplitude of the dominant harmonic, $\xi_{\max }^{s}$, and its edge value, $\xi_{\text {edge }}^{s}$, is used as a measure for the free-boundary nature. The essentially $m=5, n=-4$ perturbation

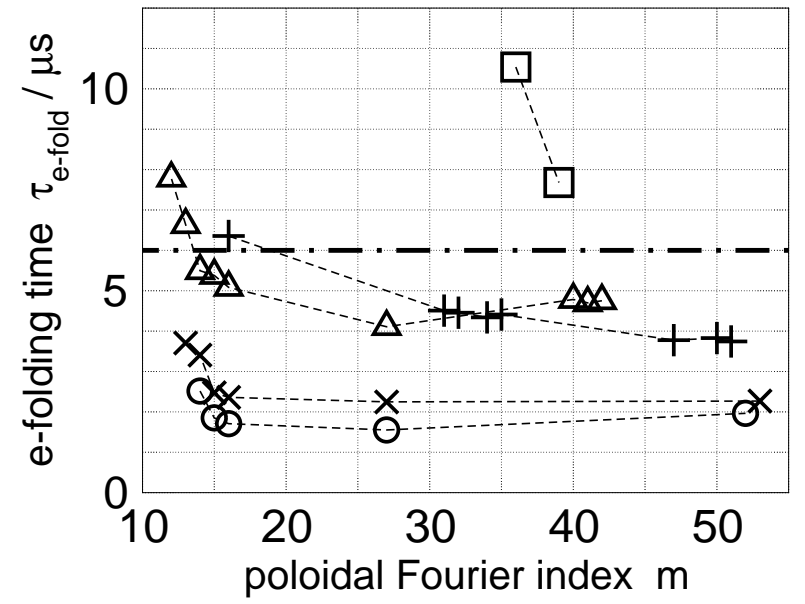

Figure 7. $e$-folding times versus dominant poloidal Fourier index $m$ for unstable MHD eigenmodes in the standard highmirror case of figure $3,\langle\beta\rangle=0.05$ and s-linear pressure $(\times)$, 。 for pressure with flattened regions, $\Delta$ for s-bell-shaped pressure. + for s-linear pressure at $\langle\beta\rangle=0.04$, $\square$ for s-linear pressure at $\langle\beta\rangle=0.03$. All perturbation mode families $(N=0,1,2)$ were used. For $\langle\beta\rangle=0.05$, the Alfvén time is indicated, too (- - - ), $\tau_{\mathrm{A}} \approx 6 \mu \mathrm{s}$ (for $B_{0}=2 \mathrm{~T}$ and $L_{\mathrm{c}}=15 \mathrm{~m}$, the field-aligned extent of the perturbation extrema on the plasma boundary in figure 10). The dashed lines are only meant to lead the eye.

of figure 6 with an e-folding time of $13 \mu$ s is manifestly free-boundary, with $\xi_{\max }^{s} / \xi_{\text {edge }}^{s} \approx 1.1$. The e-folding time of the dominantly $m=10, n=-8$ perturbation is smaller by a factor of 1.7 , and its radial structure less free-boundary with $\xi_{\max }^{s} / \xi_{\text {edge }}^{s} \approx 1.3$. At $\tau_{\mathrm{e}-\text { fold }}=$ $4 \mu \mathrm{s}$, the essentially $m=25, n=-20$ perturbation is comparatively close to being fixed-boundary with $\xi_{\text {max }}^{s} / \xi_{\text {edge }}^{s} \approx 4.5$. Consistently, when forced to be fixed-boundary, the latter perturbation does not change significantly, its e-folding time increases by $7.5 \%$. The manifestly free-boundary perturbations are stable when subjected to the fixed-boundary condition.

\subsection{Edge-rotational transform $5 / 5$}

For the standard high-mirror configuration of figure 3 , the results of the CAS3D global free-boundary stability analyses are summarized in figure 7 , which shows $e$-folding times for unstable eigenmodes versus the dominant poloidal Fourier index $m$ of the respective normal displacement harmonics. For each poloidal $m$, the most unstable eigenmode was selected. For the conversion of the CAS3D eigenvalues, a central electron number density of $n_{\mathrm{e}}(0)=310^{20} / \mathrm{m}^{3}[16,27]$ in a hydrogen plasma was used for $\langle\beta\rangle=0.05$, $n_{\mathrm{e}}(0) /\left(10^{20} / \mathrm{m}^{3}\right)=2.13$ and 1.4 for $\langle\beta\rangle=0.04$ and 0.03 , respectively.

In the standard high-mirror cases, the ideal MHD eigenmodes are resonant near the plasma boundary, i.e. the dominant harmonics approximately fulfill the 


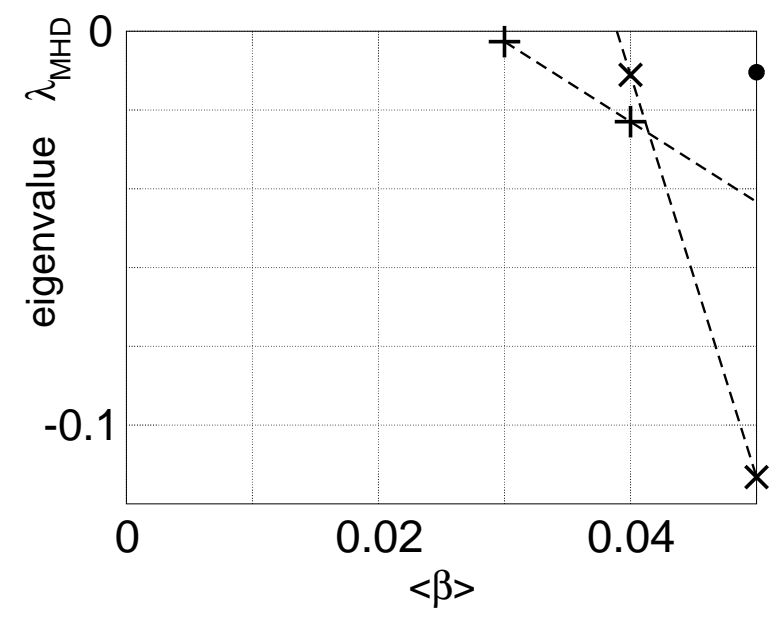

Figure 8. Ideal MHD eigenvalues versus averaged plasma- $\beta$ for the standard high-mirror case with magnetic mirror 0.11 and $s$ linear pressure. Dominant poloidal Fourier perturbation index $m=35(+) ; m=16(\times) ; m=12(\bullet)$. All perturbation mode families $(N=0,1,2)$ were used.

resonance condition, $m \iota_{\text {boundary }}+n=0$, which is typical for peeling modes [31]. In W7-X, the rotational transform is roughly unity and, for this reason, free from low-order rationals, other than the divertorrelevant edge values of $5 / 4,5 / 5$, and $5 / 6$. Hence, only relatively high mode numbers can be resonant inside the plasma. In the standard high-mirror cases studied here, the lowest dominant poloidal Fourier indices $m$ of unstable eigenmodes are, indeed, $\mathcal{O}(10)$, e.g. $(m, n)=(11,-10)$ or $(m, n)=(12,-11)$, as can be seen in figures 7 to 10 .

For $\langle\beta\rangle=0.05$, the influence of the pressure profile was studied with a linear dependence on the flux label $s$, shown as solid line in figure 4, and a bell-shaped dependence, indicated by the dashed line. The latter is more peaked near the magnetic axis and flatter near the plasma boundary. The $e$ folding times are smaller in the equilibrium with $s$ linear pressure $(x$ in figure 7 ) than the ones in the case with $s$-bell-shaped pressure $(\Delta)$. The flattening of the pressure around natural resonances, eliminating otherwise diverging parallel current densities (see section 2 and figure 4), causes more pronounced gradients elsewhere. Consequently, the $e$-folding times are larger in the equilibrium without flattened regions, $\times$ in figure 7 , as compared to the case with pressureflattening $(O)$, both for $\langle\beta\rangle=0.05$. This underlines the destabilizing nature of steeper pressure gradients, especially near the plasma edge. The small change in e-folding time, $\delta \tau_{\mathrm{e} \text {-fold }} \approx 1 \mu \mathrm{s}$, however, justifies the usage of equilibria in which pressure flattening was not invoked, but in which the components of the equilibrium parallel current density diverging at the natural resonances have been constrained to zero.
For both pressure profiles as well as for varying plasma- $\beta$ using the $s$-linear profile $[\times(+, \square)$ for $\langle\beta\rangle=0.05(0.04,0.03)$ in figure 7$]$ medium-modenumber perturbations, $m \lesssim 13$, grow on larger timescales as the high-mode-number ones do, $m \approx 25$. Hence, stability limits in terms of the averaged plasma$\beta$ depend on the perturbation mode numbers. Figure 8 shows the extrapolation of unstable MHD eigenvalues corresponding to $e$-folding times via (3) to points of marginal plasma- $\beta$. It may be concluded that the W7-X standard case is stable against low-mode-number modes, i.e. perturbation with poloidal Fourier index $m<10$. Medium-mode-number instabilities, $m \approx 10$, can exist at $\langle\beta\rangle=0.05$, which is the envisaged MHDstability limit [28]. Only pressure-driven MHD modes with higher mode-numbers may have more restrictive stability limits, e.g. $\langle\beta\rangle \approx 0.04$ for $15 \lesssim m \lesssim 20$.

Evaluation of the Alfvén time shows that all the instabilities summarized in figures 7 and 8 develop on an Alfvén time-scale. With

$\tau_{\mathrm{A}}=\frac{L_{\mathrm{c}}}{v_{\mathrm{A}}}=\frac{L_{\mathrm{c}}}{B_{0}} \sqrt{\mu_{0} \rho_{0}(0)}$

it is $\tau_{\mathrm{A}} \approx 6 \mu \mathrm{s}$ for $\langle\beta\rangle=0.05, B_{0}=2 \mathrm{~T}, n_{\mathrm{e}}(0)=$ $310^{20} / \mathrm{m}^{3}$, and a characteristic length of $L_{\mathrm{c}}=15 \mathrm{~m}$.

The latter is derived from the spatial structure of the dominantly $(m, n)=(12,-11)$ unstable eigenmode with $\tau_{e-\text { fold }} \approx 8 \mu \mathrm{s}$ at $\langle\beta\rangle=0.05$, shown in figures 9 and 10 by way of example. With many Fourier harmonics of comparable amplitude and the extrema of the normal displacement on the outside of the plasma torus, the ballooning nature is apparent. In total, the perturbations can be characterized as ballooningpeeling.

The dominant harmonics which are coupled by stellarator-type helical equilibrium components each have tokamak-type side-bands, e.g. toroidicity-induced ones. Radially, the perturbation is strongly localized near the plasma boundary. On the plasma boundary, the normal displacement forms five field-aligned stripes of strong inward and outward shift, on the outside of the torus, with an approximate length $L_{\mathrm{c}}=15 \mathrm{~m}$. Whereas in the so-called triangular cross-sections, $\varphi=$ $(36+k 72)^{\circ}, k=0, \ldots 4$, the region of strongest normal displacement is located near the tip of the triangle pointing towards the outside of the torus. In the beanshaped cross-sections, $\varphi=(0+k 72)^{\circ}, k=0, \ldots 4$, the regions of strong displacement are halfway between the outside and the tip of the cross-section.

\section{Conclusions}

In continuation and extension of earlier work [9], W7-X divertor equilibria have been computationally established and studied for their global ideal MHD properties. 


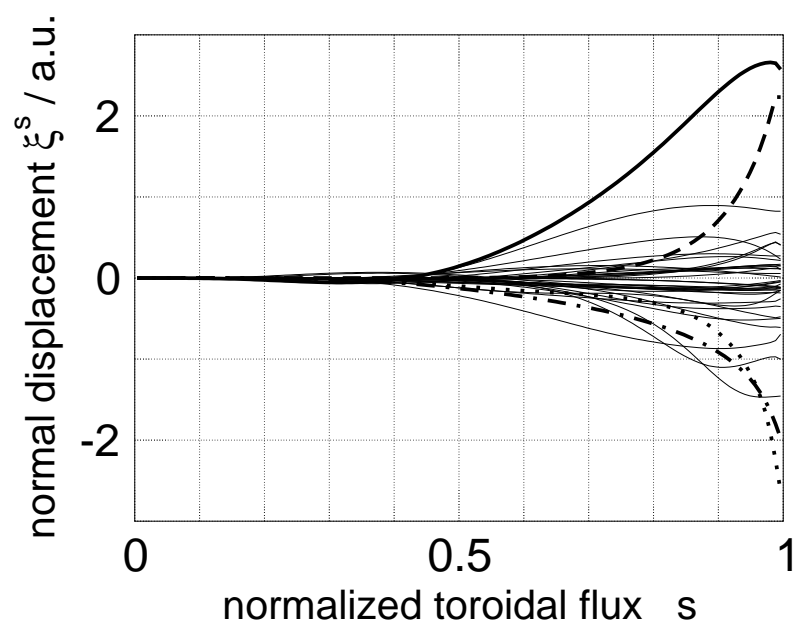

Figure 9. Fourier harmonics of the non-dimensional normal displacement, $\xi^{s}$, versus normalized toroidal flux, $s$, of the unstable odd-parity perturbation with $\tau_{e-\text { fold }} \approx 8 \mu$ s in the standard high-mirror case of figure $3,\langle\beta\rangle=0.05$, s-bell-shaped pressure. The dominant harmonics are $m=12, n=-11$ ( - ), $m=15, n=-14(\cdots \cdots), m=26, n=-24(---)$, and $m=17, n=-16(-\cdot-)$. CAS3D computation parameters: 325 perturbation Fourier harmonics (40 strongest shown), 128 radial points.
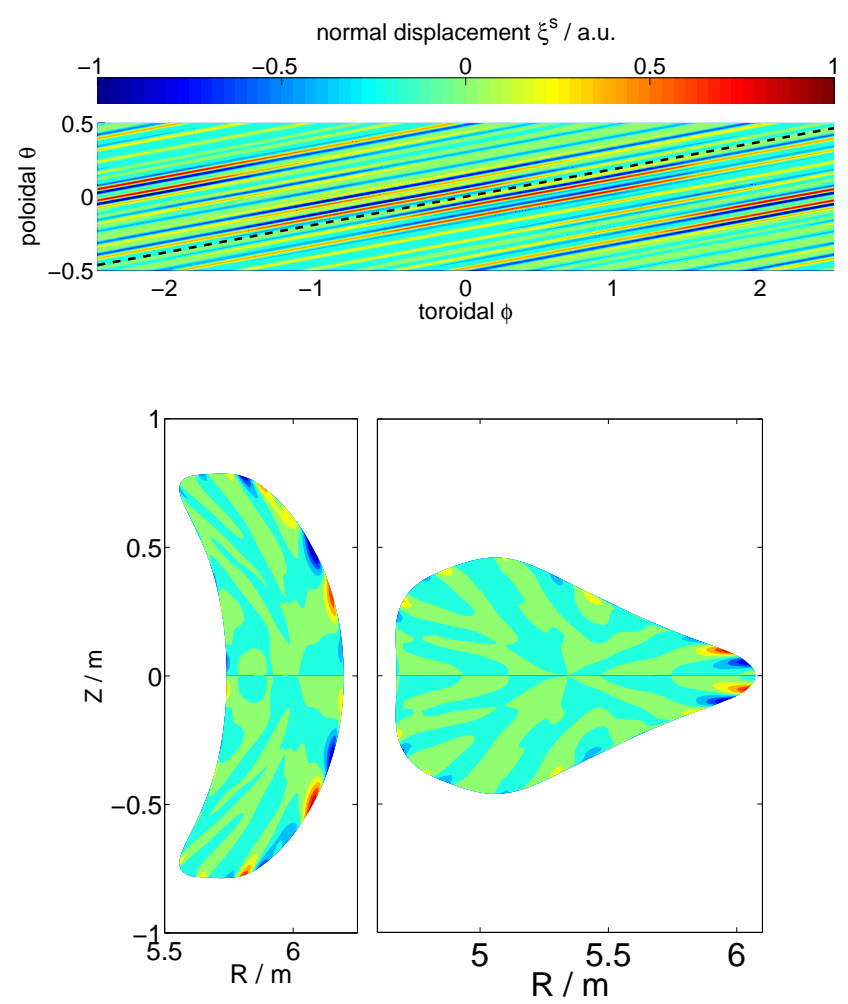

Figure 10. Unstable, odd-parity perturbation in the standard high-mirror case of figure $3,\langle\beta\rangle=0.05$, and figure 9: Contours of the normal displacement, $\xi^{s}$ in magnetic coordinates, on the developed plasma boundary (compare figure 6), on the $\varphi=0^{\circ}$ bean-shaped, and on the $\varphi=180^{\circ}$ triangular cross-section. One toroidal transit of the magnetic field-line $(\iota=0.93)$ is indicated as --- .
In the low-iota case with the $5 / 6$ island for the divertor at the plasma edge, unstable low-mode number, free-boundary perturbations exist even at low plasma- $\beta$. With essentially one dominant Fourier harmonic and small side-bands, they are Merciertype global modes. In a low-shear net-currentfree stellarator, this is consistent with the lack of shear stabilization and vacuum magnetic well, the local Mercier criterion thus being violated. Using equilibrium pressure and mass-density profiles as well as the central value of the equlibrium mass density from a transport analysis, $e$-folding times of $\mathcal{O}(10) \mu \mathrm{s}$ are found. If studied experimentally in W7-X, this scenario might clarify the question whether a vacuum magnetic well is needed for MHD stability of smallshear stellarators. A relaxation of this requirement would have a direct impact on the magnetic geometry by possibly making the strong indentation dispensable, which forms the inboard side of the torus in the beanshaped cross section.

The standard case with the $5 / 5$ islands at the plasma edge is stable against low-mode-number modes, poloidal $m<10$, for the foreseen value of the magnetic mirror, $m_{\text {ratio }}=0.1$. Since, for $\langle\beta\rangle \gtrsim 0.04$, ballooningtype, medium-mode-number, unstable free-boundary perturbations exist according to the computational global mode analysis, this W7-X scenario might be useful to experimentally study the question whether MHD modes with poloidal $m \gtrsim 10$ might affect the plasma edge region of small-shear stellarators in a dangerous way.

This work will be continued with global ideal MHD stability studies of divertor equilibria with bootstrap current and, eventually, equilibria reconstructed from experimental W7-X data.

\section{Acknowledgments}

This work has been carried out within the framework of the EUROfusion Consortium and has received funding from the European Union's Horizon 2020 research and innovation programme under grant agreement number 633053. The views and opinions expressed herein do not necessarily reflect those of the European Commission.

\section{Appendix A. Coil currents}

The W7-X magnet system consists of fifty modular and twenty planar coils. The five-fold periodicity and the stellarator symmetry of the machine reduce to five modular coil type and two planar ones. Additionally, there are ten in-vessel divertor coils and five largedimension correction coils outside the cryostat. The divertor and correction coils are not used in this work. 
Table A1. Current loads for the W7-X variants discussed in this work. The cases are identified by the rotational transform on the plasma edge, $\iota_{\text {edge }}$, and by the magnetic mirror on the magnetic

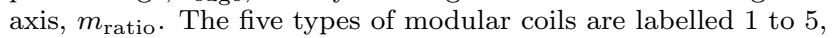
the two types of planar coils are A and B.

\begin{tabular}{|c|c|c|c|c|c|c|}
\hline \multicolumn{5}{|c|}{$I_{\text {modular }} / \mathrm{MA}$} & \multicolumn{2}{|c|}{$I_{\text {planar }} / \mathrm{MA}$} \\
\hline 1 & 2 & 3 & 4 & 5 & A & B \\
\hline $\mathrm{a} 1.4294$ & 0.9884 & 0.9817 & 0.9566 & 0.5422 & 0.1186 & 0.4454 \\
\hline b 1.404 & 1.3506 & 1.2261 & 1.1794 & 1.111 & 0.007 & 0.007 \\
\hline
\end{tabular}

In table $\mathrm{A} 1$, the current loads are summarized which define the W7-X variants studied in sections 2 and 3 . With these current amplitudes, the central magnetic field strength is $B_{\text {axis }} \approx 2 \mathrm{~T}$.

\section{References}

[1] Weller A, Anton M, Geiger J, Hirsch M, Jaenicke R, Werner A, W7-AS Team, Nührenberg C, Sallander E and Spong D A 2001 Phys. Plasmas 8931

[2] Weller A, Geiger J, Werner A, Zarnstorff M C, Nührenberg C, Sallander E, Baldzuhn J, Brakel R, Burhenn R, Dinklage A, Fredrickson E, Gadelmeier F, Giannone L, Grigull P, Hartmann D, Jaenicke R, Klose S, Knauer J P, Könies A, Kolesnichenko Y I, Laqua H P, Lutsenko V V, McCormick K, Monticello D, Osakabe M, Pasch E, Reiman A, Rust N, Spong D A, Wagner F, Yakovenko Y V, W7-AS Team and NBI-Group 2003 Plasma Phys. Control. Fusion 45 A285

[3] Weller A, Sakakibara S, Watanabe K Y, Toi K, Geiger J, Zarnstorff M C, Hudson S R, Reiman A, Werner A, Nührenberg C, Ohdachi S, Suzuki Y, Yamada H, W7-AS Team and LHD Team 2006 Fusion Science and Technology $\mathbf{5 0} 158$

[4] de Aguilera A M, Castejón F, Ascasibar E, Blanco E, la Cal E D, Hidalgo C, Liu B, López-Fraguas A, Medina F, Ochando M A, Pastor I, Pedrosa M A, Milligen B V, Velasco J L and the TJ-II Team 2015 Nucl. Fusion 55 113014

[5] Sakakibara S, Watanabe K, Takemura Y, Okamoto M, Ohdachi S, Suzuki Y, Narushima Y, Ida K, Yoshinuma M, Tanaka K, Tokuzawa T, Yamada I, Yamada H and Takeiri Y 2015 Nucl. Fusion 55083020

[6] Gasparotto M, Baylard C, Bosch H S, Hartmann D, Klinger T, Vilbrandt R and Wegener L 2014 Fusion Engineering and Design 892121

[7] Pedersen T S, Andreeva T, Bosch H S, Bozhenkov S, Effenberg F, Endler M, Feng Y, Gates D, Geiger J, Hartmann D, Hlbe H, Jakubowski M, Knig R, Laqua H, Lazerson S, Otte M, Preynas M, Schmitz O, Stange T, Turkin Y and the W7-X Team 2015 Nucl. Fusion 55 126001

[8] Boscary J, Stadler R, Peacock A, Hurd F, Vorköper A, Mendelevitch B, Cardella A, Pirsch H, Tittes H, Tretter J, Li C, Greuner H and Smirnow M 2011 Fusion Engineering and Design 86572

[9] Nührenberg C 1996 Phys. Plasmas 32401

[10] Bernstein I B, Frieman E A, Kruskal M D and Kulsrud R M 1958 Proc. Roy. Soc. Ser. A 24417

[11] Hain K, Lüst R and Schlüter A 1957 Z. Naturforsch. 12a 833

[12] Herrnegger F 1987 Z. Naturforsch. 42a 1085
[13] Nakamura Y, Ichiguchi K, Wakatani M and Johnson J L 1989 J. Phys. Soc. Japan 583157

[14] G Y Fu, W A Cooper, R Gruber, U Schwenn, and D V Anderson 1992 Phys. Fluids B 41401

[15] Schwab C 1993 Phys. Fluids B 53195

[16] Geiger J, Beidler C D, Feng Y, Maaßberg H, Marushchenko N B and Turkin Y 2015 Plasma Phys. Control. Fusion 57014004

[17] Strumberger E and Schwarz E 2005 Vacfield code: Computation of the vacuum magnetic field and its first derivatives for various coil types Tech. Rep. IPP 5/112 MPI Plasmaphysik Garching, Germany http://hdl.handle.net/11858/00-001M-0000-002716A7-C

[18] Hirshman S P and Whitson J C 1983 Phys. Fluids 263553

[19] Hirshman S P, van Rij W I and Merkel P 1986 Comput. Phys. Commun. 43143

[20] Nührenberg J and Zille R 1986 Phys. Lett. A 114129

[21] Nührenberg C, Boozer A H and Hudson S R 2009 Phys. Rev. Lett. 102235001

[22] Loizu J, Hudson S R, Bhattacharjee A, Lazerson S and Helander P 2015 Phys. Plasmas 22090704

[23] Gourdon G, Marty D, Maschke E and Dumont J 1969 Proceedings of the 3rd International Conference on Plasma Physics Controlled Nuclear Fusion Research, Novosibirsk, USSR, 1968 (Nucl. Fusion Suppl. vol 1) (Vienna: International Atomic Energy Agency) p 847

[24] Drevlak M, Monticello D and Reiman A 2005 Nucl. Fusion 45731

[25] Shafranov V and Zakharov L 1972 Nucl. Fusion 12599

[26] Strumberger E 1997 Nucl. Fusion 3719

[27] Turkin Y, Geiger J, Maaßberg H, Beidler C D and Marushchenko N B 2014 41st EPS Conf. on Contr. Fus. and Plasma Phys., Berlin 2014 URL http://ocs.ciemat.es/EPS2014PAP/pdf/P1.086.pdf

[28] Grieger G, Beidler C D, Maaßberg H, Harmeyer E, Herrnegger F, Junker J, Kisslinger J, Lotz W, Merkel P, Nührenberg J, Rau F, Sapper J, Schlüter A, Sardei F and Wobig H 1989 Plasma Physics and Controlled Nuclear Fusion Research 1990 (Nucl. Fusion Suppl. vol 2) (Vienna: International Atomic Energy Agency) p 369

[29] Mercier C 1962 Plasma Physics and Controlled Nuclear Fusion Research 1961 (Nucl. Fusion Suppl. vol 2) (Vienna: International Atomic Energy Agency) p 801

[30] Nührenberg J and Zille R 1988 Theory of Fusion Plasmas Varenna 1987 (Bologna: Società Italiana di Fisica) p 3 ISBN 8877940093

[31] Lortz D 1975 Nucl. Fusion 1549

[32] Correa-Restrepo D 1978 Z. Naturforsch. 33a 789 